\title{
Analysis of Vertical Walking Force of Single Footstep
}

\author{
Wang Caifeng ${ }^{a^{*}}$, Gao Shiqiao ${ }^{b}$, Niu Shaohua ${ }^{c}$, Liu Haipeng ${ }^{d}$, Tan Yangkang ${ }^{e}$ \\ School of Mechatronical Engineering, Beijing Institute of Technology, Beijing, China \\ arudygey@126.com, bgaoshq@bit.edu.cn, cshh@bit.edu.cn, dihp@bit.edu.cn, \\ etanyangkang@163.com
}

Keywords: Walking; Contact time; Correlation; Shape amplitude; Impulse

Abstract. The traditional vertical walking force model does not take account of the variations of intro-subject and inter-subject, this paper aims at analyzing the characteristics of vertical walking force of single footstep. Using a test platform, two single footstep traces are collected. Force shape parameters including characteristic time and shape amplitudes, as well as contact time and normalized impulse of single footstep are investigated. The contact time and normalized impulse are all fitted as functions of step frequency. Finally, based on the statistical results, a new concept equivalent force is put forward to describe the single footstep walking force.

\section{Introduction}

Interest has been aroused to investigate the dynamic loads due to walking because of excessive human-induced vibrations of footbridges $[1,2,3]$. The traditional vertical walking force model is perfectly periodic and it is represented as a sum of Fourier harmonics. However, this model ignores the variability of successive footsteps in real walking. The left footstep and right footstep have different walking parameters from a long observation of human walking, while these differences can cause notable departure between real walking forces and perfectly periodic loads [4]. The narrow band randomness of real walking forces indicates that the old-fashioned model needs to be revised to meet the demands of designing and predicting in engineering [5]. Hence, it is very necessary to investigate the single footstep walking force and obtain its characteristics.

The work in this paper is aimed at providing fundamental data and giving a basis to motivate the research for human walking forces. The outline is as follows. Section 2 briefly describes the experimental measurements. In Section 3, the walking force shape parameters of single footstep, including characteristic time and shape amplitude, as well as contact time and normalized impulse are dealt with, the variability between continuous walking forces of single footsteps is presented. Finally a few inherent drawbacks of the experiment design i.e. reasons causing errors are discussed.

\section{Experimental Measurement}

The platform test system is composed of two force plates, an amplifier, a data acquisition and a laptop, as exhibited in Fig. 1. The force plate consists of a rigid aluminum top plate and four built-in piezoelectric trial-component force sensors arranged at four corners of the plate. The two force plates are mounted on the rigid floor lengthways, ensuring in the same horizontal plane. The two plates named A and B are staggered with a space between them, so it is easy for the test subject to walk freely from the anterior plate to the posterior plate.

The experiments are carried out in a wide space in the laboratory. Before formal test, the test subject needs to stand still on the force plate for $3 \mathrm{~s}$ to get static weight. When the test begins, the test subject needs to walk $2 \mathrm{~m}$ (leading-in) before setting foot on plate A and continue to walk $1 \mathrm{~m}$ (leading-out) after leaving plate B with a preferred speed, which ensure the walking as realistically 


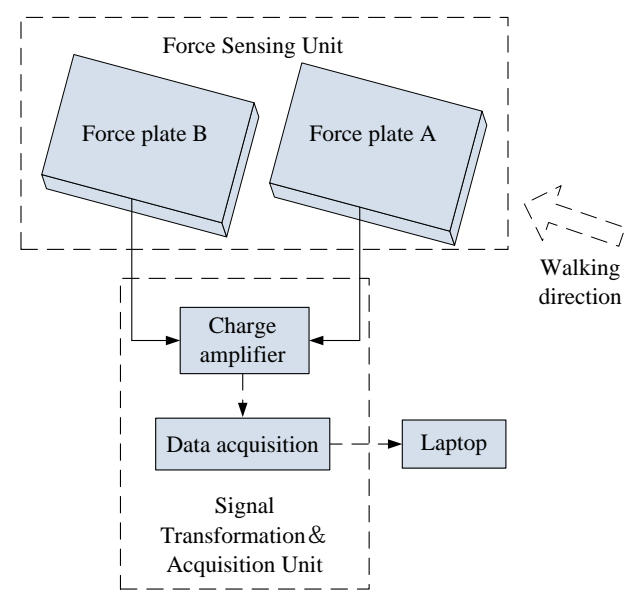

Fig. 1. Schematic diagram of platform test system.

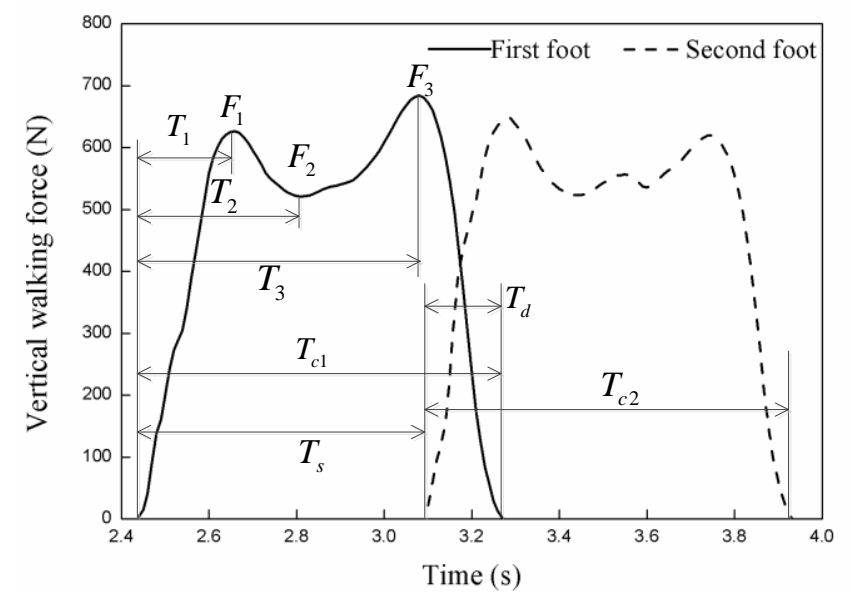

Fig. 2. Vertical force trajectories of two continuous Footsteps.

as possible. Each test subject repeats the test for three times. Note that the test subject can step onto the force plate with a freely chosen left or right foot. Altogether 7 healthy adults (4 males and 3 females, including one foreigner) are invited to participate in the experiment, with body mass $59.9 \pm 16.1 \mathrm{Kg}$, age $26.0 \pm 1.9$ years, height $170.2 \pm 12.3 \mathrm{~cm}$. The sample rate is set as $1000 \mathrm{~Hz}$. At least two footsteps can be monitored.

\section{Results and Discussion}

Two typical vertical force trajectories of continuous walking are presented in Fig. 2. A complete step is defined to begin with the heel-strike-ground moment and ends with the next heel-strike-ground moment [6], the duration is step period $\left(T_{s}\right)$, also known as single-foot-stance-phase time, while the left time is the double-feet-stance-phase time $\left(T_{d}\right)$, obviously the step frequency is

$$
f_{s}=\frac{1}{T_{s}} .
$$

where $T_{s}=T_{c 1}-T_{d}$.

Force Shape. As shown in Fig. 2 the force shape feature is characterized by three local extrema and corresponding characteristic time. The three shape amplitudes, $F_{1}, F_{2}$ and $F_{3}$ are the most remarkable dynamic contribution with corresponding characteristic time $T_{1}, T_{2}$ and $T_{3}$ respectively. Fig. 3 illustrates a scatter of characteristic time (divided by contact time) and shape amplitudes (divided by weight). The characteristic time varies between reasonable ranges for $T_{1}[0.14,0.27]$, $T_{2}$ [0.36, 0.54], $T_{3}[0.71,0.80]$ separately, where $T_{2}$ has the largest dispersion, $T_{3}$ has the least. Usually the dynamic load amplitudes $F_{1}$ and $F_{3}$ exceed one, i.e. larger than static body weight due to the push of the foot, while $F_{2}$ is less than 0.90 due to the swing of the body. As shown in Fig. 4, significant correlations are obtained with positive correlation with step frequency for $F_{1}$ and $T_{2}$, negative correlation for $T_{1}$ and $F_{2}$. It is indicated that when $f_{s}$ becomes larger, $T_{1}$ minishes, accordingly $F_{1}$ increases, while $T_{2}$ increases, $F_{2}$ minishes. The small correlation coefficient of $F_{3}$ means it has weak relationship with step frequency. 


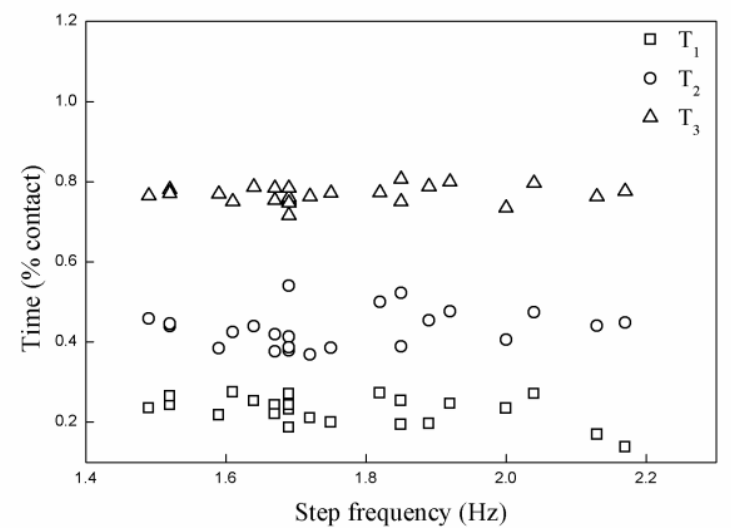

(a)

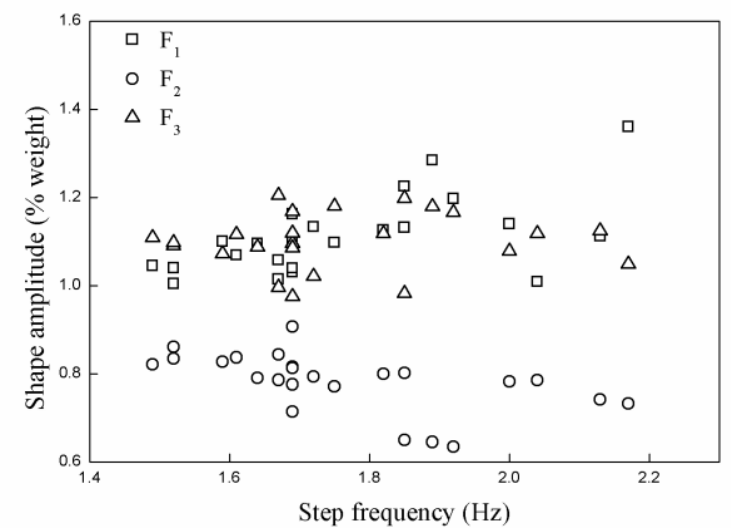

(b)

Fig. 3. Scatter diagram of characteristic time and shape amplitudes: (a) characteristic time, (b) shape amplitudes.

Examples of force shapes are given for the special situation with the same step frequency $1.69 \mathrm{~Hz}$, as seen in Fig. 5(a). The weight normalized force trajectories vary among different persons, even for the same person (B1 and B2). The force shapes of left and right footstep in each test are very similar but still have slight differences, implying the imperfections of force parameters between the left and right footstep. Obviously the diversity of the curves cannot be reflected in the conventional model relying only on step frequency.

Contac Time. The contact time of single footstep is researched hereunder. The contact time of left footstep is not inevitably the same with that of the right footstep. The differences between left and right footstep suggest that there is strong leg and weak leg as reported by Kasperski [7]. Fig. 5(b) gives five sets of data at the same step frequency $1.69 \mathrm{~Hz}$. The slight variations predict that in walking process the contact time of the supporting foot as well as the double-feet-stance-phase time, with left and right foot constantly alternating, is always changing under various circumstances for different people (even same person), revealing the randomness of locomotion of walking.

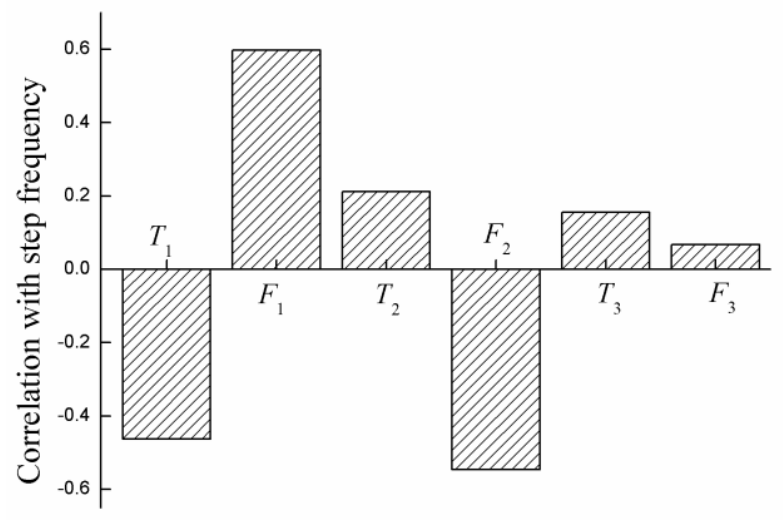

Fig. 4. Correlation coefficients with step frequency for characteristic time and shape amplitudes. 


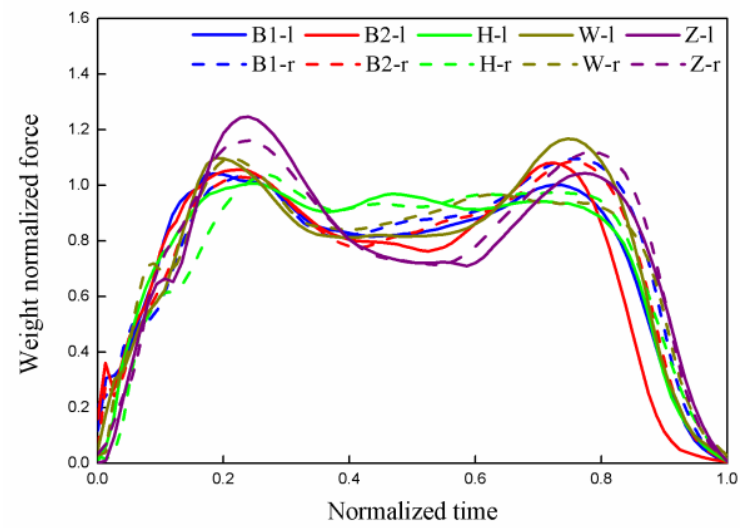

(a)

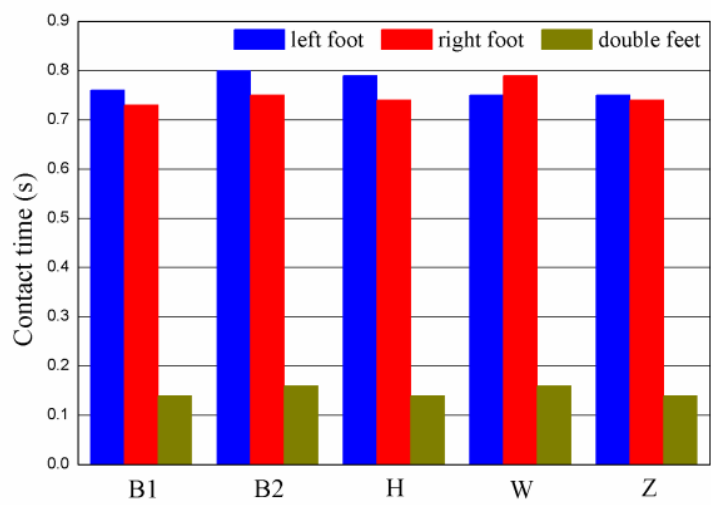

(b)

Fig. 5. Comparison of force trajectories and contact time at the same frequency of $1.69 \mathrm{~Hz}$ (a) force trajectories, left footstep (solid line), right footstep (dash line), different color represents different test subject (B1 and B2 represent two distinct test data for the same person B). (b) contact time, left foot (blue), right foot (red), double feet (dark yellow).

Despite of the indeterminacy and randomness, the data shows that the contact time of single footstep has an approximately reciprocal relation with step frequency as illustrated in Fig. 6, which is fitted as

$$
T_{c}=1.269 f_{s}^{-1.019} \text {. }
$$

The statistical results show that the duration of double-feet-stance-phase is about $20.59 \%$ of the entire contact time of single footstep, which is smaller than the result according to $\mathrm{Li}$ [8]. This may due to the unease that the test subject feels on the force plate, so the test subject has to be cautious to take a step.

Footfall Impulse and Equivalent Force. The footfall impulse of single footstep is calculated by the integral of walking force over contact time. Results of all the weight normalized impulses for left and right footstep are shown in Fig. 7. The apparent declining tendency offers an opportunity to describe normalized impulse as a function of step frequency, which is

$$
I_{n}=0.9499 f_{s}^{-0.9384} \text {. }
$$

where $I_{n}$ represents weight normalized impulse. It is noteworthy that the obvious trend for normalized impulse to reduce with increasing step frequency is logical, nevertheless, it has a larger scatter than expected.

In order to reduce the variability of intro-subject and inter-subject as far as possible, a more deterministic parameter for single footstep should be searched. Hence, the concept equivalent force is put forward, defined as weight normalized impulse divided by contact time, i.e.

$$
F_{e}=\frac{I_{n}}{T_{c}}
$$

Bearing in mind that the dimensionless parameter $F_{e}$ quantitatively represents the averaged weight normalized force during the contact time of single footstep. Coefficient of variation (CoV), defined as standard deviation divided by mean, is used to validate the feasibility of equivalent force. $\mathrm{CoV}$ along with standard deviation (SD) and mean (M) of the shape amplitude, contact time, normalized impulse and equivalent force are listed in Table 1. 


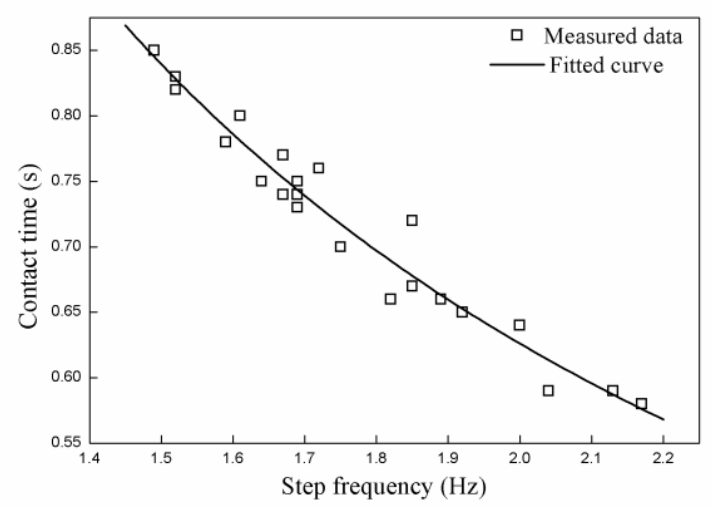

Fig. 6. Measured contact time of first foot.

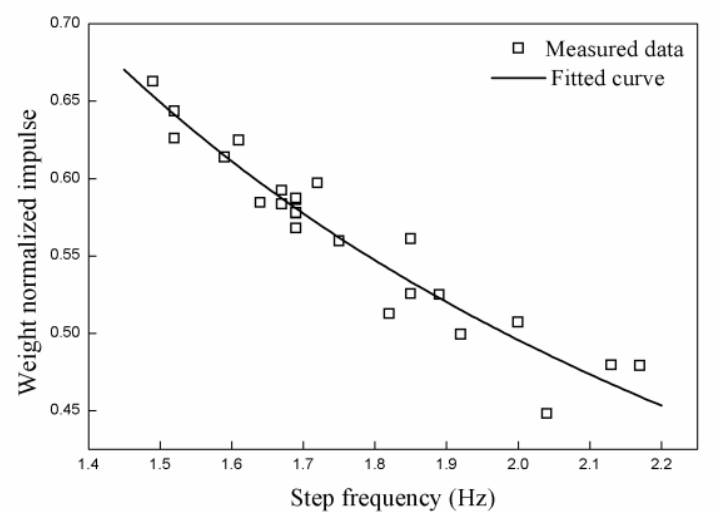

Fig. 7. Weight normalized footfall impulses.

The CoV values for contact time and normalized impulse are obviously larger than the work by Brownjohn [9], however, the lowest $\mathrm{CoV}$ value of equivalent forces indicates that the equivalent force changes least compared with shape amplitude, contact time and normalized impulse, which means that the equivalent force has the minimum influence from different test subjects and step frequencies. The equivalent force containing the least variability of intro-subject and inter-subject is more accurate and reliable, it is very useful to construct a more precise force model containing continuous footsteps, and also it does good to analyze the vertical walking forces of single pedestrian and even crowd during a period of time.

A few shortcomings in the design of the experimentation should be pointed out. Due to the limitation of experiment, only two successive footsteps corresponding to one step frequency are recorded in each test. Although the vertical height of the force plate is too small to be ignored, it is still harmful because actually it may compel the test subject to adjust the gait to make 'step-in' or 'step-out' action, which does not agree with the real situation of natural walking.

Table 1. Comparison of SD, $\mathrm{M}$ and $\mathrm{CoV}$ for shape amplitude (only $F_{1}$ ), contact time, normalized impulse and equivalent force.

\begin{tabular}{cccc}
\hline Parameter & Standard deviation & Mean & CoV \\
\hline Shape amplitude & 0.089 & 1.114 & $7.99 \%$ \\
Contact time & 0.081 & 0.731 & $11.08 \%$ \\
Normalized impulse & 0.056 & 0.565 & $9.91 \%$ \\
Equivalent force & 0.016 & 0.784 & $2.04 \%$ \\
\hline
\end{tabular}

\section{Conclusions}

Walking forces of two continuous single footsteps are collected by the test platform. Force shape parameters, including characteristic time and shape amplitude, along with contact time and weight normalized impulse are analyzed. The strong correlation indicates that with step frequency increasing, the first shape amplitude increases while the first characteristic time lessens, meanwhile the second shape amplitude decreases and the second characteristic time grows. At the same step frequency the contact time and force shapes of left and right footstep have slight variation, verifying the randomness and imperfections of intro-subject and inter-subject. Then power functions are used to describe the contact time and normalized impulse versus step frequency. In the end a new concept equivalent force is presented, which is a dimensionless parameter with the minimal 
influences from different subjects and step frequencies. The work in this paper provides much fundamental information for constructing vertical walking forces of a sequence of footsteps involving the imperfections of intro-subject and inter-subject. It is hopeful to serve as a framework for establishing a more thorough and realistic model of continuous vertical walking forces.

\section{References}

[1] S. Zivanovic, A. Pavic, P. Reynolds, Vibration serviceability of footbridges under human-induced excitation: a literature review, Journal of Sound and Vibration 279 (2005) 1-74.

[2] E.T. Ingólfsson, C.T. Georgakis, J. Jönsson, Pedestrian-ind uced lateral vibrations of footbridges: a literature review, Engineer Structures 45 (2012) 21-52.

[3] S.Q. Gao, D. Wang, L. Jin, Influence of the human body on the characteristics of a pedestrian brid ge, 18th ISSAT International Conference on Reliability and Quality in Design. US A, 2012.

[4] V. Racic, J.M.W. Brownjohn, Stochastic model of near-periodic vertical loads due to humans walking, Advanced Engineering Informatics 25 (2011) 259-275.

[5] C. Sahnaci, M. Kasperski, Prediction of the vibrations of pedestrian structures under random pedestrian streams, 9th International Conference on Structural Dynamics, Portugal, (2014) 1065-1072.

[6] V. Racic, A. Pavic, J.M.W. Brownjohn, Experimental identification and analytical modelling of human walking forces: literature review, Journal of Sound and Vibration 326 (2009) 1-49.

[7] C. Sahnaci, M. Kasperski, Random loads induced by walking, Sixth European Conference on Structural Dynamics EURODYN, Paris, (2005) 441-446.

[8] Q. Li, J.S. Fan, J.G. Nie, Q.W. Li, C. Y, Crowd-induced random vibration of footbridge and vibration control using multiple tuned mass dampers, Journal of Sound and Vibration 329 (2010) 4068-4092.

[9] J.M.W. Brownjohn, A. Pavic, P. Omenzetter, A spectral density approach for modelling continuous vertical forces on pedestrian structures due to walking, Canadian Journal of Civil Engineering 31 (2004) 65-77. 